\title{
Non-Radiative Recombination of Triplet Charge- Transfer State as the Key of Limiting Efficiency Mediates the Positive Relevance of JSC and VOC
}

\author{
Yue Ren \\ Yanbian University \\ Ming-Yang Li \\ Yanbian University \\ Yun Geng \\ Northeast Normal University \\ Yi-Zhou Xu \\ Yanbian University \\ Guang-Yan Sun ( $\square$ gysun@ybu.edu.cn ) \\ Yanbian University https://orcid.org/0000-0002-5236-9753 \\ Ming-Yue Sui \\ Yanbian University \\ Zhong-Min Su \\ Northeast Normal University https://orcid.org/0000-0002-3342-1966
}

\section{Article}

Keywords: Organic solar cell, non-fullerene acceptor, triplet charge-transfer state, non-radiative recombination, time-dependent density functional theory

Posted Date: September 28th, 2021

DOI: https://doi.org/10.21203/rs.3.rs-942912/v1

License: (c) (1) This work is licensed under a Creative Commons Attribution 4.0 International License. Read Full License 


\title{
Non-Radiative Recombination of Triplet Charge-Transfer State as the Key of Limiting Efficiency Mediates the Positive Relevance of $J_{\mathrm{SC}}$ and $V_{\mathrm{OC}}$
}

Yue Ren, ${ }^{a}$ Ming-Yang Li, ${ }^{a}$ Yun Geng, ${ }^{b}$ Yi-Zhou Xu, ${ }^{a}$ Guang-Yan Sun, ${ }^{* a}$ Ming-Yue Sui ${ }^{* a}$ and Zhong-Min Su ${ }^{a, b}$

a. Department of Chemistry, Faculty of Science, Yanbian University, Yanji 133002, Jilin, P.

R. China; Email: gysun@ybu.edu.cn

b. Institute of Functional Material Chemistry, Faculty of Chemistry, Northeast Normal University, Changchun 130024, Jilin, P. R. China

\begin{abstract}
Non-fullerene organic solar cells (NF OSCs) with A-D-A acceptors have realized the positive relevance of short-circuit current density $\left(J_{\mathrm{SC}}\right)$ and open-circuit voltage $\left(V_{\mathrm{OC}}\right)$, because of the restricted energy loss. However, non-radiative energy loss remains unclear, resulting in the positive relevance could not maximize power conversion efficiency (PCE). Here, the impact of non-radiative recombination directly related to the singlet- and triplet-charge-transfer $\left({ }^{1} \mathrm{CT}\right.$ and ${ }^{3} \mathrm{CT}$ ) states on the positive relevance is explored. It establishes the essential connection between ${ }^{3} \mathrm{CT}$-state non-radiation and positive relevance, points out the former mainly hinders PCE. The root reason is that decisive factors of decay rates in two pathways are completely different, but hard to adjust coordinately. Especially, another trade-off is still detected in NF OSCs, causing a bottleneck in PCE. To the end, we propose the defects of A-D-A molecular design by revealing ${ }^{3} \mathrm{CT}$-state non-radiation mediates the positive relevance.
\end{abstract}

\section{Keywords}

Organic solar cell, non-fullerene acceptor, triplet charge-transfer state, non-radiative 
recombination, time-dependent density functional theory

\section{Introduction}

As a promising technology, the power conversion efficiency (PCE) of state-of-the-art organic solar cells (OSCs) with donor: acceptor bulk heterojunction structure has surpassed $18 \% .{ }^{1-3}$ High-efficiency charge separation is achieved at almost negligible energy offset in OSCs based on non-fullerene acceptors (NFAs) with A-D-A structure. ${ }^{4-6}$ The trade-off inherent in fullerene-OSCs between short-circuit current density $\left(J_{\mathrm{SC}}\right)$ and open-circuit voltage $\left(V_{\mathrm{OC}}\right)$ that affects PCE, is addressed. ${ }^{7,8}$ Furthermore, the realization of positive relevance could simultaneously increase $J_{\mathrm{SC}}$ and $V_{\mathrm{OC}}$ among the NFA systems with A-D-A structure. ${ }^{7,9}$ However, such phenomenon has not yield the highest PCE. Meanwhile, PCE is still limited by energy loss mediated by charge-transfer (CT) state. ${ }^{10,11}$ It is crucial to understand the impact of energy loss on the positive relevance of $J_{\mathrm{SC}}$ and $V_{\mathrm{OC}}$.

Compared with significant driving force produced by fullerene systems with weak absorption and weak emission properties, non-radiative energy loss $\left(\Delta E_{3}\right)$ is the main cause of energy loss in NFA systems with high-efficient charge separation at low driving force. ${ }^{12}$ The generation of $\Delta E_{3}$ is shown in Fig. 1(a), singlet-CT $\left({ }^{1} \mathrm{CT}\right)$ state is allowed to decay to the ground state (GS) $\left({ }^{1} \mathrm{CT} \rightarrow \mathrm{GS}\right)$ or convert to the triplet-CT $\left({ }^{3} \mathrm{CT}\right)$ state $\left({ }^{1} \mathrm{CT} \rightarrow{ }^{3} \mathrm{CT}\right)$ in geminate recombination process. ${ }^{13}$ The previous studies almost tend to focus on ${ }^{1} \mathrm{CT}$-state non-radiation and improve this mechanism, which can effectively avoid non-radiative loss by promoting ${ }^{1} \mathrm{C}$ T state luminescence. ${ }^{14}$ But $\Delta E_{3}$ is still insurmountable and further restricts $V_{\mathrm{OC}}$ because of they ignore the potential effect of the ${ }^{3} \mathrm{CT}$ non-radiation, where the complicated processes of ${ }^{3} \mathrm{CT}$ state decay to $\mathrm{GS}\left({ }^{3} \mathrm{CT} \rightarrow \mathrm{GS}\right)$ or convert into triplet local exciton $\left({ }^{3} \mathrm{LE}\right)$ states of either donor or acceptor $\left({ }^{3} \mathrm{CT} \rightarrow{ }^{3} \mathrm{LE}\right) .{ }^{15,16}$ The previous studies almost tend to focus on the ${ }^{1} \mathrm{CT}$-state non-radiation, but ignore the influence of the ${ }^{3} \mathrm{CT}$ non-radiation. As a result, the potential effect of ${ }^{3} \mathrm{CT}$-state recombination on $\Delta E_{3}$ remains unclear.

In this study, the fundamental mechanism of $\Delta E_{3}$ on positive correlation of $J_{\mathrm{SC}}$ and $V_{\mathrm{OC}}$, are explored by a multi-scale method, along with the ${ }^{1} \mathrm{CT} \rightarrow \mathrm{GS}$ and ${ }^{3} \mathrm{CT} \rightarrow{ }^{3} \mathrm{LE}$ pathways. The results demonstrate that the decay rates tracing the two pathways are respectively controlled by different factors. ${ }^{3} \mathrm{CT} \rightarrow{ }^{3} \mathrm{LE}$ pathway mainly determines the $\Delta E_{3}$, and thus affects the positive relevance. Importantly, we prove that the root reason is potential existence of trade-off, which makes it 
difficult to obtain the maximum PCE for positive correlation in NF OSCs. Our research establishes the internal connection between the $\Delta E_{3}$ mediated by ${ }^{3} \mathrm{CT}$ state and the positive relevance of $J_{\mathrm{SC}}$ and $V_{\mathrm{OC}}$, and finally proposes the drawback in the molecular design of A-D-A structure from the perspective of energy loss.

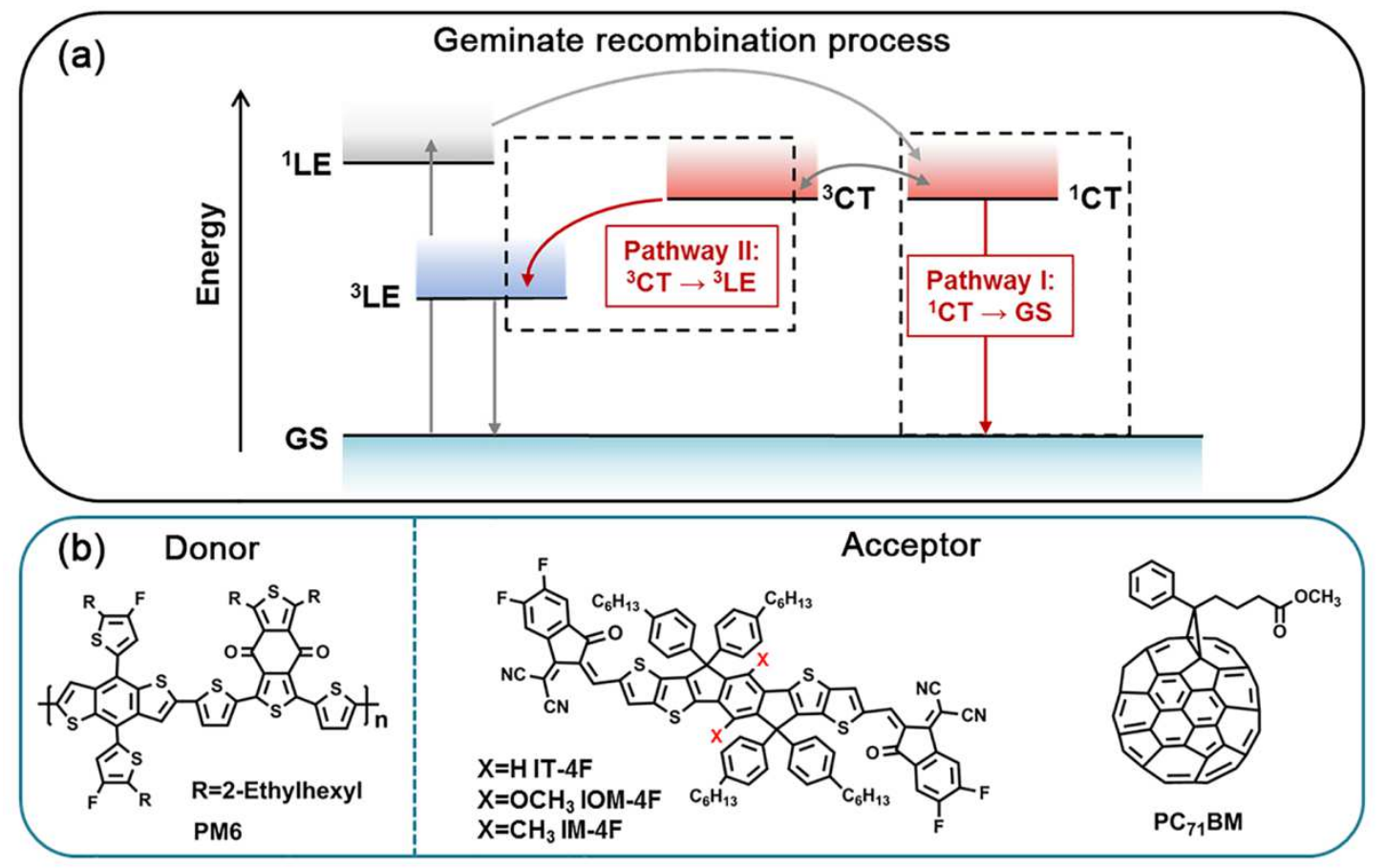

Fig. 1 (a) Schematic diagram of geminate recombination process including singlet charge transfer $\left({ }^{1} \mathrm{CT}\right)$ state decay to ground state $(\mathrm{GS})\left({ }^{1} \mathrm{CT} \rightarrow \mathrm{GS}\right)$ and triplet charge transfer $\left({ }^{3} \mathrm{CT}\right)$ state convert to triplet local exciton $\left({ }^{3} \mathrm{LE}\right)$ state $\left({ }^{3} \mathrm{CT} \rightarrow{ }^{3} \mathrm{LE}\right)$, where ${ }^{3} \mathrm{CT}$ state is formed by ${ }^{1} \mathrm{CT}$ state via intersystem crossing. (b) Chemical structures of non-fullerene IT-4F, IOM-4F, IM-4F, fullerene $\mathrm{PC}_{71} \mathrm{BM}$ as acceptors and PM6 as donors.

\section{Models and Methods}

\section{Models}

IT-4F, IOM-4F, and IM-4F with A-DD'D-A structure were select as research objects, which $J_{\mathrm{SC}}$ and $V_{\mathrm{OC}}$ of the three NFA-based blends presented a gradient change of positive relevance (Fig. 1(b) and Supplementary Table 1). ${ }^{8}$ The differences of structures were reflected on the D' unit of the A-DD'D-A structure, where the hydrogen atom of IT-4F on D' unit is replaced by methoxy group 
for IOM-4F and methyl group for IM-4F severally. ${ }^{8}$ For purposes of comparison, fullerene derivative $\mathrm{PC}_{71} \mathrm{BM}$ was used as reference with a negative relevance of $J_{\mathrm{SC}}$ and $V_{\mathrm{OC}}$ in fullerene-based blend. ${ }^{17}$ All systems were based on the same donor copolymer PM6, which was simplified as four units because the good convergence of the HOMO energy levels (see Supplementary Fig. 1).

\section{Methods}

\section{Molecular dynamics simulation details}

Molecular dynamics (MD) simulation were performed using the GROMACS 2018.4 software package. ${ }^{18}$ Atom types and intra- and inter-molecular interaction parameters of PM6 and acceptors were built from the general AMBER force field (GAFF) ${ }^{19}$ and charges were obtained by the restricted electrostatic potential (RESP) fitting method. ${ }^{20}$ The simulations were carried out under three-dimensional periodic boundary conditions by means of the leap-frog integrator. A spherical cut-off of $1.0 \mathrm{~nm}$ for summation of Van de Waals interactions and the Particle-Mesh-Ewald (PME) method for long-range Coulomb interactions were employed throughout. ${ }^{21}$ All equilibration processes were as follows: (i) Randomly placing 200 molecules in the periodic box size of $100 \AA$ $\times 100 \AA \times 100 \AA$ for PM6/acceptors to generate an initial geometry; (ii) 2 fs of NVT ensemble and $1 \mathrm{~ns}$ of NPT ensemble to make molecules close together. In the NPT ensemble, pressure and temperature were hold at constant 1 bar and $300 \mathrm{~K}$ adopted Parrinello-Rahman barostat ${ }^{22}$ and the velocity rescaling thermostat, ${ }^{23}$ respectively. (iii) 150 ns of equilibration were conducted at NPT ensemble to obtain better equilibrium conformations.

\section{Quantum chemical calculation}

The radiative recombination rate $\left(k_{\mathrm{r}}\right)$ of ${ }^{1} \mathrm{CT}$ state is obtained from the Einstein coefficient relation: ${ }^{24}$

$k_{\mathrm{r}}=\frac{\left(E_{\mathrm{CT}}\right)^{3} f(\mathrm{n})}{3 \varepsilon_{0} \pi h^{4} c^{3}}\left|\mu_{\mathrm{tr}}\right|^{2}$

where $\mu_{\mathrm{tr}}$ is the transition dipole moment; $\varepsilon_{0}$ is the vacuum permittivity; $h$ is the Planck constant; and $c$ is the speed of light in vacuum. In classical Marcus electrons hopping model, ${ }^{25}$ non-radiative recombination rate $\left(k_{\mathrm{nr}}\right)$ of ${ }^{1} \mathrm{CT}$ and ${ }^{3} \mathrm{CT}$ state is defined as: $: 26,27$ 
$k_{\mathrm{nr}}=\frac{2 \pi}{h}\left|V_{\mathrm{el}}\right|^{2} \frac{1}{\sqrt{2 \sigma^{2}}} \exp \left(-\frac{\left(\lambda-E_{\mathrm{CT}}\right)^{2}}{2 \sigma^{2}}\right)$

where $V_{\text {el }}$ denotes the electronic coupling between initial and final states; $\sigma$ is the static energy disorder; and $\lambda$ is the reorganization energy. Three-state model was applied to account for the electronic couplings and reorganization energy between the GS, CT and LE states, and the calculation details were given in the Supporting Information. Three-state model was applied to account for the electronic couplings, reorganization energy, and other parameters in the decay rate.

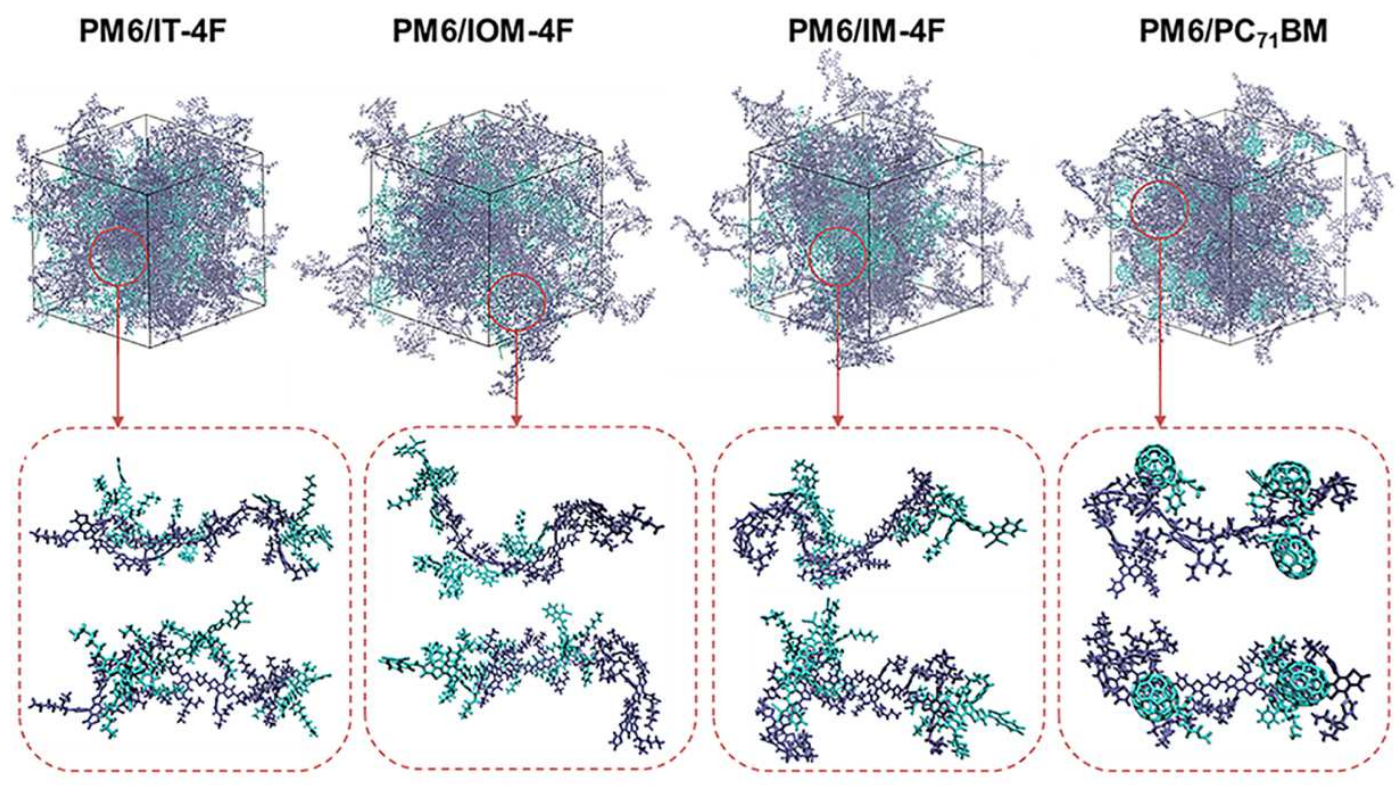

Fig. 2 Molecular clusters of PM6/acceptor blends in molecular dynamics (MD) simulations.

For monomers, the PM6 was simplified into two repeated units and the side chains were substituted based on extracted molecular clusters, because of the HOMO and LUMO mainly delocalized on two repeat units. ${ }^{28}$ Donor PM6 and all acceptors were optimized at the PBE0/6-31G(d) levels, because of PBE0 method could provide an accurate evaluation of geometric and electronic structures for thiophene derivatives. ${ }^{29,30}$ The lowest ${ }^{3} \mathrm{LE}$-state energies were evaluated at TD-PBE0/6-31G(d) levels. For PM6/acceptor blends, the simulation results include a variety of packing modes, among which the face-on orientation is favorable for exciton dissociation. Through the statistics of face-on configuration, the typical PM6/acceptor molecular clusters and blending morphologies were extracted from the final 5 ns (Fig. 2 and Supplementary 
Fig. 2). The lowest ${ }^{1} \mathrm{CT}$ - and ${ }^{3} \mathrm{CT}$-state energies and decay rates were calculated at the TD-CAM-B3LYP/6-31G(d) levels. ${ }^{31}{ }^{1} \mathrm{CT}$ and ${ }^{3} \mathrm{CT}$ state were identified by the natural transition orbital (NTO) method described by Multiwfn 3.7, that is, the electron is located on the acceptor, the hole is located on the donor (Supplementary Fig. 3). ${ }^{32}$ All the above-mentioned calculations were performed in the Gaussian 09 D.01 software package. ${ }^{33}$

\section{Results and discussion}

\section{Radiative and non-radiative recombination originated from ${ }^{1} \mathrm{CT} \rightarrow \mathrm{GS}$ pathway}

${ }^{1} \mathrm{CT} \rightarrow \mathrm{GS}$ pathway suffers losses via radiative and non-radiative recombination. ${ }^{34}$ In order to estimate the impact of ${ }^{1} \mathrm{CT} \rightarrow \mathrm{GS}$ loss pathway on $\Delta E_{3}$ under the positive relevance of $J_{\mathrm{SC}}$ and $V_{\mathrm{OC}}$ of NFA systems, ${ }^{1} \mathrm{CT}$-state energy and decay rate are worth exploring. After all, the energy could illustrate the proportion of $\Delta E_{3}$, which directly affect the positive relevance. Based on the result of the energy arrangement, the decay rate could further indicate that the trajectory and time of $\Delta E_{3}$ contributed from ${ }^{1} \mathrm{CT} \rightarrow \mathrm{GS}$ loss pathway.

As we all know, the CT-state energy is related to the energy level of the acceptor, with constant donor. Along with the change on D' unit of IT-4F $\rightarrow \mathrm{IOM}-4 \mathrm{~F} \rightarrow \mathrm{IM}-4 \mathrm{~F},{ }^{1} \mathrm{CT}$-state energy increases gradually with $2.11 \rightarrow 2.41 \rightarrow 2.96 \mathrm{eV}$, which is associated with positive correlation (Supplementary Table 1 and 2). Therefore, we speculate modifying the D' unit according to the electron-donating/withdrawing character, may achieve the simultaneous increase of $J_{\mathrm{SC}}$ and $V_{\mathrm{OC}}$.

In terms of the decay rate of ${ }^{1} \mathrm{CT}$ state, for a fullerene system with weak luminescence and weak absorption, it is difficult to reduce the energy loss even if $k_{\mathrm{r}}^{1 \mathrm{CT}}$ is higher than $k_{\mathrm{nr}}^{1 \mathrm{CT}}$ in PM6/PC ${ }_{71} \mathrm{BM}$. However, NFA with strong absorption and strong emission could lead to large $E Q E_{\mathrm{EL}}$ values, since $E Q E_{\mathrm{EL}}$ affected $k_{\mathrm{r}}$ and $k_{\mathrm{nr}}$, is in an inverse relationship with $\Delta E_{3}$ (detailed equation (1) and (2) in Supporting Information). From PM6/IT-4F to PM6/IOM-4F, as shown in Fig. 3, $k_{\mathrm{r}}^{\mathrm{I}} \mathrm{CT}$ is increased from $8.87 \times 10^{3} \mathrm{~s}^{-1}$ to $3.02 \times 10^{5} \mathrm{~s}^{-1}$, following a significant downward trend of $k_{\mathrm{nr}}^{1} \mathrm{CT}$ by three orders, leading a decrease of $\Delta E_{3}$. The increase of $k_{\mathrm{r}}^{1 \mathrm{CT}}$ is mainly attributed to increasing ${ }^{1} \mathrm{CT}$-state energy and $\mu_{\mathrm{tr}}$. For $k_{\mathrm{nr}}^{1 \mathrm{CT}}$, the smaller substituent group on D' unit has little effect on the $\sigma$. Although $V_{\text {el }}$ increases, the decrease of $k_{\mathrm{nr}}^{1 \mathrm{CT}}$ is caused by increasing the value of 
exponential term, i.e., the difference between $\lambda$ and ${ }^{1} \mathrm{CT}$-state energy. As we all know, the difference between $\lambda$ and ${ }^{1} \mathrm{CT}$-state energy is close to 0 , which can maximize value of $k_{\mathrm{nr}}^{1}$. It indicates that $V_{\mathrm{el}}$ has little effect compared to the parameter in the exponential term just under ${ }^{1} \mathrm{CT} \rightarrow \mathrm{GS}$ pathway. From PM6/IOM-4F to PM6/IM-4F, $\Delta E_{3}$ has not been reduced although the $k_{\mathrm{r}}^{1} \mathrm{CT}$ and $k_{\mathrm{nr}}^{1 \mathrm{CT}}$ present the downward trend overall. Among the factors of $k_{\mathrm{r}}^{1} \mathrm{CT}, \mu_{\mathrm{tr}}$ is observably decreased because of the decrease of oscillator strength. A downward trend of $k_{\mathrm{r}}^{1} \mathrm{CT}$ is presented from $3.02 \times 10^{5} \mathrm{~s}^{-1}$ to $7.11 \times 10^{2} \mathrm{~s}^{-1}$, even if ${ }^{1} \mathrm{CT}$-state energy increases. Moreover, the 10 -fold drop in $k_{\mathrm{nr}}^{1 \mathrm{CT}}$ is still originated from the increase in exponential terms. However, the fact is just the opposite, the positive relevance of $J_{\mathrm{SC}}$ and $V_{\mathrm{OC}}$ still appears. Furthermore, the three orders of magnitude decline in the value of $k_{\mathrm{r}}^{1} \mathrm{CT}$ is significantly less than the eleven orders of magnitude decline of $k_{\mathrm{nr}}^{1 \mathrm{CT}}$, which likewise leads to decreased $\Delta E_{3}$.

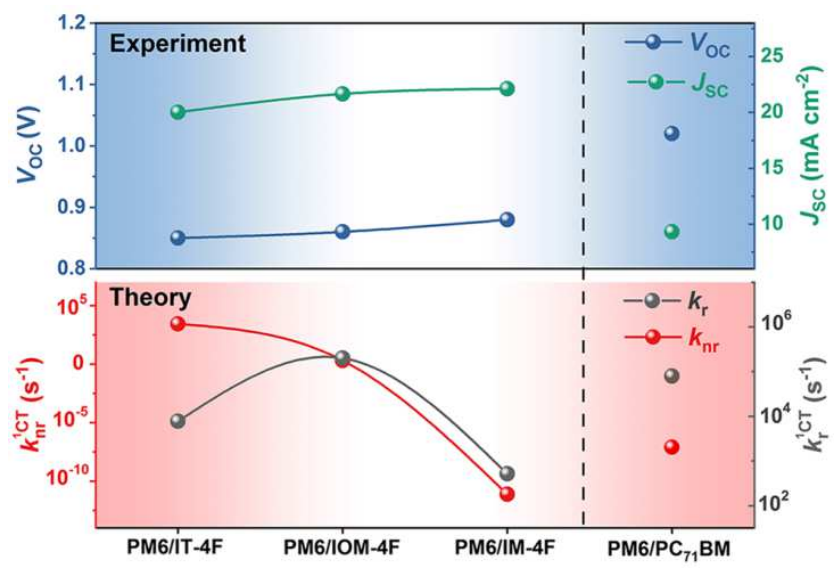

Fig. 3 Experimental $J_{\mathrm{SC}}$ and $V_{\mathrm{OC}}$ and theoretical radiative and non-radiative recombination rates $k_{\mathrm{r}}^{1} \mathrm{CT}$ and $k_{\mathrm{nr}}^{1 \mathrm{CT}}$ of ${ }^{1} \mathrm{CT} \rightarrow \mathrm{GS}$ pathway (from Ref. 8).

In two cases, although the trends of $k_{\mathrm{r}}^{1} \mathrm{CT}$ and $k_{\mathrm{nr}}^{1} \mathrm{CT}$ are different, both lead to a decrease in $\Delta E_{3}$, and further achieve simultaneous increase of $J_{\mathrm{SC}}$ and $V_{\mathrm{OC}}$. Meanwhile, it laterally reflects that remaining the A-DD'D-A backbone structure of NFAs unchanged, and increasing ${ }^{1}$ CT-state energy could indeed reduce the loss of ${ }^{1} \mathrm{CT} \rightarrow \mathrm{GS}$ pathway to achieve positive relevance. To comprehensively consider the impact of non-radiative recombination, another ${ }^{3} \mathrm{CT} \rightarrow{ }^{3} \mathrm{LE}$ loss 
pathway is worth further exploration.

\section{Non-radiative recombination originated from ${ }^{3} \mathrm{CT} \rightarrow{ }^{3} \mathrm{LE}$ pathway}

Compared with ${ }^{1} \mathrm{CT} \rightarrow \mathrm{GS}$ pathway, ${ }^{3} \mathrm{CT} \rightarrow \mathrm{GS}$ is almost ignored because of forbidden transition, ${ }^{3} \mathrm{CT} \rightarrow{ }^{3} \mathrm{LE}$ route is more complicated. ${ }^{35}$ Experimentally, it is intricate to detect ${ }^{3} \mathrm{CT}$ signal directly, and further to distinguish the ${ }^{1} \mathrm{CT}$ - and ${ }^{3} \mathrm{CT}$-state energies, but is realizable for theoretical study. ${ }^{36}$ As depicted in Fig. 4(a) and Supplementary Table 3 the ${ }^{1} \mathrm{CT}$ - and ${ }^{3} \mathrm{CT}$-state energies are degenerate, which are consistent with many results, proving the accuracy of our calculations. According to the attribution of ${ }^{3} \mathrm{LE}$, it could be divided into ${ }^{3} \mathrm{CT} \rightarrow{ }^{3} \mathrm{LE}$ and ${ }^{3} \mathrm{CT} \rightarrow{ }^{3} \mathrm{LE}$, affected by the energy of ${ }^{3} \mathrm{LE}$ state and decay rate. For energy, since the ${ }^{3} \mathrm{LE}$ state of PM6 is closer to ${ }^{3} \mathrm{CT}$ state than that of NFAs, ${ }^{3} \mathrm{CT} \rightarrow{ }^{3} \mathrm{LE}_{\mathrm{D}}$ may be inclined to exist mainly. Improving ${ }^{3} \mathrm{LE}$ state of donor to increase the capability of transition again to ${ }^{3} \mathrm{CT}$ state could promote photocurrent generation. In this case, NFAs as variables seem not to affect the loss of ${ }^{3} \mathrm{CT} \rightarrow{ }^{3} \mathrm{LE}$ pathway. Here speculates if ${ }^{3} \mathrm{LE}$-state energy of NFA is close to ${ }^{3} \mathrm{CT}$-state energy, it should have a more favorable impact on the improvement of photovoltaic parameters, because the conversion of singlet-triplet state may easily occur, accompanied by more complicated situations.
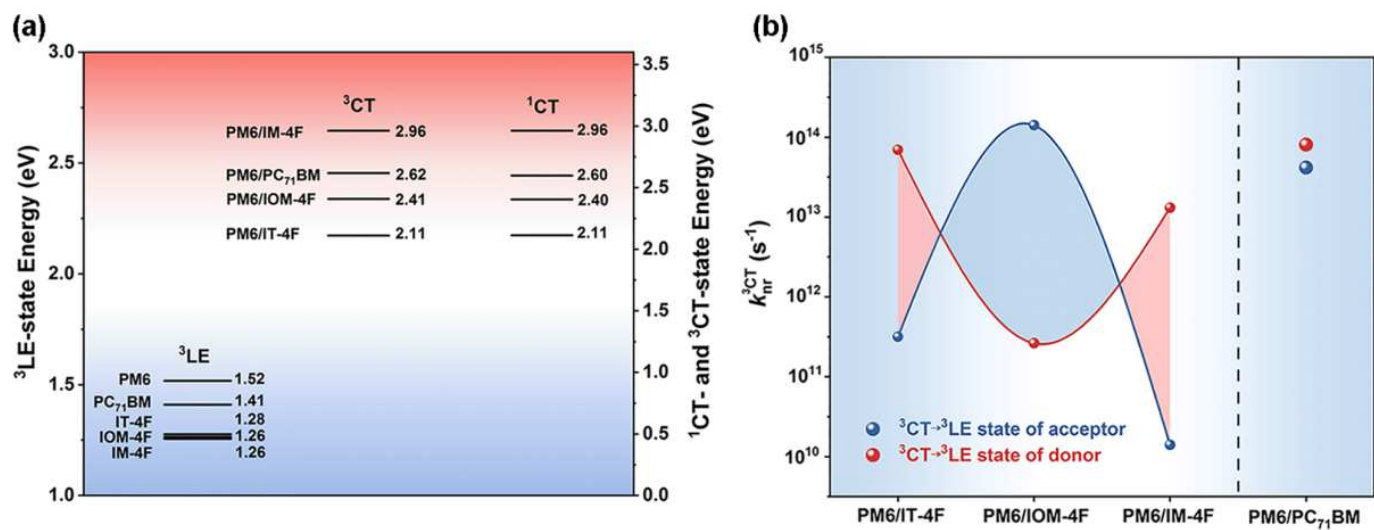

Fig. 4 (a) Schematic diagram of ${ }^{3}$ LE-states, interfacial ${ }^{1} \mathrm{CT}$ - and ${ }^{3} \mathrm{CT}$-states energies (eV) for

PM6/acceptors blends. (b) The change curve of $\left.k_{\mathrm{nr}}^{3} \mathrm{CT}^{-1}\right)$, corresponding to decay from ${ }^{3} \mathrm{CT}$ states to ${ }^{3} \mathrm{LE}$ state of donor and acceptors.

To complement the results of ${ }^{3} \mathrm{CT} \rightarrow{ }^{3} \mathrm{LE}$ pathway based on energy alignments, the decay rates of ${ }^{3} \mathrm{CT}$ state are calculated, where the higher value of rate determine the trend of pathway possibly. 
Fig. 4(b), Supplementary Table 4 and 5 provide a comparison of decay probability to ${ }^{3}$ LE state between donor and acceptor. For $\mathrm{PM} 6 / \mathrm{PC}_{71} \mathrm{BM}$, two similar values of $k_{\mathrm{nr}}^{3 \mathrm{CT}}$ determine the larger

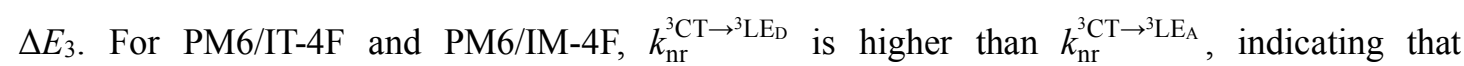
${ }^{3} \mathrm{CT} \rightarrow{ }^{3} \mathrm{LE}$ pathway plays a major role in the process. Inversely for $\mathrm{PM} 6 / \mathrm{IOM}-4 \mathrm{~F}$, the ${ }^{3} \mathrm{CT} \rightarrow{ }^{3} \mathrm{LE}_{\mathrm{A}}$ pathway takes the lead, on account of the fluctuation of $V_{\text {el }}$ rather than exponential term $\left(\lambda-E_{\mathrm{CT}}\right)$. Because the decrease in the exponential term (close to 0 ) could not increase the value of $k_{\mathrm{nr}}^{3 \mathrm{CT}}$. For example, from PM6/IT-4F to PM6/IOM-4F along with ${ }^{3} \mathrm{CT} \rightarrow{ }^{3} \mathrm{LE}_{\mathrm{D}}$ pathway, $0.473 \mathrm{eV}$ of exponential term turns down $0.210 \mathrm{eV}$, but $k_{\mathrm{nr}}^{3 \mathrm{CT}}$ still declines. The reason is that $V_{\mathrm{el}}$ has dropped by 30 times. There are more variables under the ${ }^{3} \mathrm{CT} \rightarrow{ }^{3} \mathrm{LE}$ pathway, resulting in uncontrollable $k_{\mathrm{nr}}^{{ }^{3} \mathrm{CT}}$ and the absence of linear correlation between $k_{\mathrm{nr}}^{3 \mathrm{CT}}$ and positive relevance. Nevertheless, the order of magnitude of $k_{\mathrm{nr}}^{3 \mathrm{CT}}$ is significantly higher than that of $k_{\mathrm{nr}}^{1 \mathrm{CT}}$. On the premise of positive relevance, the loss of ${ }^{3} \mathrm{CT}$ state instead of ${ }^{1} \mathrm{CT}$ state mainly controls $\Delta E_{3}$, and further influences the positive relevance of $J_{\mathrm{SC}}$ and $V_{\mathrm{OC}}$.

\section{The relationship of ${ }^{1} \mathrm{CT} \rightarrow \mathrm{GS}$ and ${ }^{3} \mathrm{CT} \rightarrow{ }^{3} \mathrm{LE}$ non-radiative rate affecting $\Delta E_{3}$}

Based on the previous analysis, many differences are found between ${ }^{1} \mathrm{CT} \rightarrow \mathrm{GS}$ and ${ }^{3} \mathrm{CT} \rightarrow{ }^{3} \mathrm{LE}$ non-radiative pathway, mainly in energy and the factors that affect the decay rate. Compared to ${ }^{1} \mathrm{CT} \rightarrow \mathrm{GS}$ pathway, a larger value of $k_{\mathrm{nr}}^{3 \mathrm{CT}}$ of ${ }^{3} \mathrm{CT} \rightarrow{ }^{3} \mathrm{LE}$ pathway is obtained in the three PM6/NFAs blends (Fig. 5 and Supplementary Fig. 4). For instance in PM6/IM-4F blend, the order of magnitude of $k_{\mathrm{nr}}^{3 \mathrm{CT}}\left(1.16 \times 10^{13} \mathrm{~s}^{-1}\right.$ and $\left.1.48 \times 10^{10} \mathrm{~s}^{-1}\right)$ is larger than $k_{\mathrm{nr}}^{1 \mathrm{CT}}\left(1.15 \times 10^{-11} \mathrm{~s}^{-1}\right)$. Under each pathway, the decisive factor of $k_{\mathrm{nr}}$ and consequences are different. For ${ }^{1} \mathrm{CT} \rightarrow \mathrm{GS}$ pathway, $k_{\mathrm{nr}}^{1} \mathrm{CT}$ is dominated by energy level. Because the ground state is definite, the raised ${ }^{1} \mathrm{CT}$-state energy is generated by small changes in the structure of NFAs. Compared to the product term $\left(V_{\mathrm{el}}\right)$ with a smaller change, the exponential term $\left(\lambda-E_{\mathrm{CT}}\right)$ has a greater impact. For ${ }^{3} \mathrm{CT} \rightarrow{ }^{3} \mathrm{LE}$ pathway, the actual existence of the two pathways to ${ }^{3} \mathrm{LE}$ state of donor or acceptor complicates the impact of the interfacial triplet loss. We think $k_{\mathrm{nr}}^{3} \mathrm{CT}$ is mainly affected by $V_{\text {el. }}$. Relative to the smaller difference in exponential terms, $V_{\text {el }}$ with very large fluctuation has the greatest impact, which seems to be uncontrollable. It further causes the losses of two pathways cannot be 
controlled together through simple structural modifications.

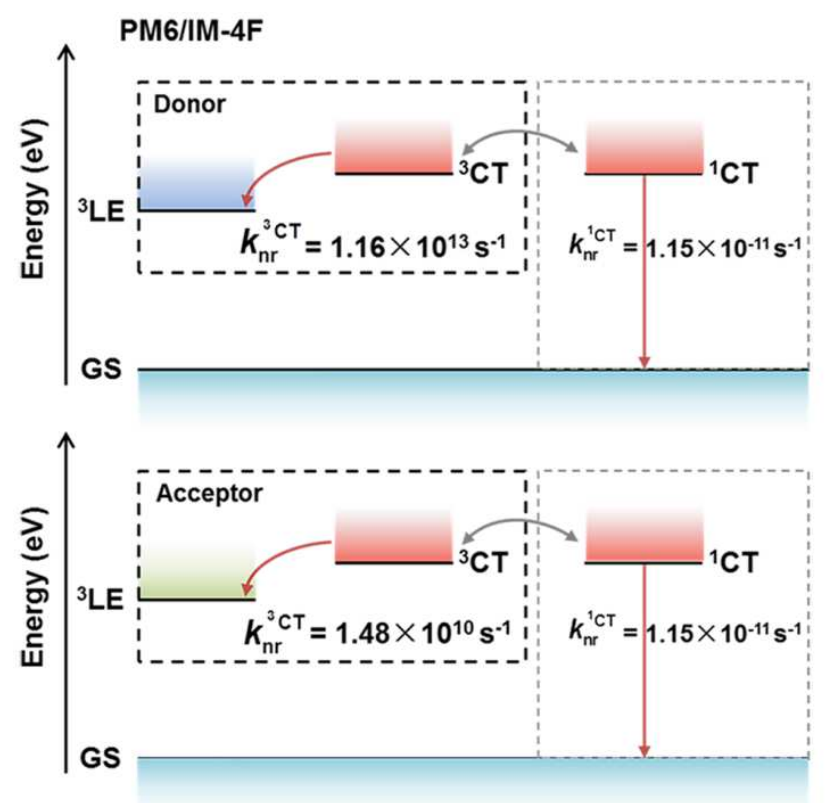

Fig. 5 Schematic diagram of non-radiative recombination rates of ${ }^{1} \mathrm{CT} \rightarrow \mathrm{GS}$ and ${ }^{3} \mathrm{CT} \rightarrow{ }^{3} \mathrm{LE}$ pathway in PM6/IM4F blend. The top is the ${ }^{3} \mathrm{CT}$ state decay to ${ }^{3} \mathrm{LE}$ state of donor, and the bottom is decay to ${ }^{3} \mathrm{LE}$ state of acceptor.

Even if the influencing factors are different, the dominant role of ${ }^{3} \mathrm{CT} \rightarrow{ }^{3} \mathrm{LE}$ pathway is confirmed in the non-radiative recombination process, whether from the energy offset or the decay rate. Furthermore, the ${ }^{3} \mathrm{CT} \rightarrow{ }^{3} \mathrm{LE}$ pathway has a major impact on the positive relevance of $J_{\mathrm{SC}}$ and

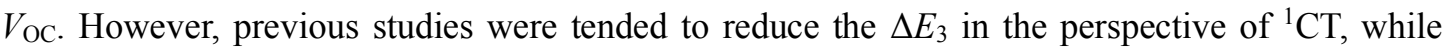
ignoring the role of ${ }^{3} \mathrm{CT}$. Based on this knowledge, we found that the realization of positive relevance leads to a slight increase in PCE (from $13.80 \%$ to $14.17 \%$ in Supplementary Table 1). It seems PCE is still restricted and low than $18 \%$. And it is unclear whether the problem arises in the interfacial triplet loss has not been suppressed.

\section{A trade-off in NF-OSCs?}

Based on above A-DD'D-A structure of NFAs, the realization of positive relevance is not equivalent to the maximum efficiency. According to the conclusions of the former sections, it is speculated that the reason is the interfacial triplet loss. Where is the bottleneck? Is it a specific 
state or a problem from fundamental A-DD'D-A structure?

To analyze the reasons for limiting PCE, the relative increase amplitude $\Delta J_{\mathrm{SC}}$ and $\Delta V_{\mathrm{OC}}$ were investigated (Fig. 6(a)). We found that there is still a trade-off in the non-fullerene systems, limiting the PCE! Specifically, although both $\Delta J_{\mathrm{SC}}$ and $\Delta V_{\mathrm{OC}}$ are greater than 0 , the increased $\Delta J_{\mathrm{SC}}$ is unexpectedly accompanied by decreased $\Delta V_{\mathrm{OC}}$. Is it a specific state or a problem from fundamental A-DD'D-A structure? To avoid data contingency, we further summarize five groups of NFA with the positive relevance of $J_{\mathrm{SC}}$ and $V_{\mathrm{OC}}$ reported (Fig. 6(b) and Supplementary Table 6). This feature is observed by calculating the slope $(k)$ of the functions, where $k<0$ in all blends (Fig. 6(c)). It most likely comes from the ${ }^{3} \mathrm{CT}$-state loss, but the root cause may be in its own structure.

(a)

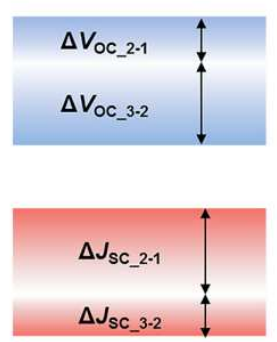

(b)

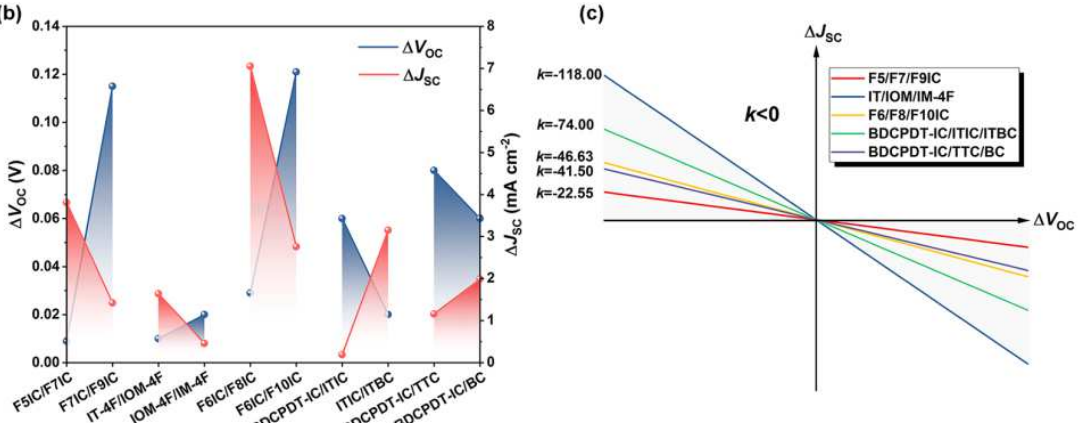

Fig. 6 (a) Schematic diagram of increment $\Delta V_{\mathrm{OC}}$ and $\Delta J_{\mathrm{SC}}$ of $V_{\mathrm{OC}}$ and $J_{\mathrm{SC}}$ measured experimentally, the numeric marker represents the position of the comparison object. (b) Calculated $\Delta V_{\mathrm{OC}}$ and $\Delta J_{\mathrm{SC}}$ for five groups of NFAs, details shown in Supplementary Table 6. (c) Calculated $\Delta J_{\mathrm{SC}}$ as a function of $\Delta V_{\mathrm{OC}}, k$ is the slope.

Faced with trade-offs, what are the differences between fullerenes and non-fullerenes? For fullerene-based blends, the trade-off is obvious and reflected in the negative relevance of $J_{\mathrm{SC}}$ and $V_{\mathrm{OC}}$, whereas the trade-off of $\Delta J_{\mathrm{SC}}$ and $\Delta V_{\mathrm{OC}}$ is internal in NFA-based blends. These findings suggest that, despite significant differences in both mechanisms and photovoltaic parameters, a trade-off consists in both fullerene and NFA systems and is difficult to be counteracted. It is reasonable to suspect that the A-DD'D-A structure of NFAs has two sides, not only brings the advantages of positive relevance, but also hides a certain degree of loss. Maybe a more rigid 
structure is required to achieve weak phosphorescent emission from ${ }^{3} \mathrm{CT}$ state to GS, to ameliorate some problems.

\section{Conclusions}

Considering the effect of non-radiative recombination loss mediated by $\mathrm{CT}$ state on positive relevance of $J_{\mathrm{SC}}$ and $V_{\mathrm{OC}}$ and thus the influence on PCE, a series of NFA systems with A-DD'D-A structure were studied. This work clarifies the leading role of the ${ }^{3} \mathrm{CT} \rightarrow{ }^{3} \mathrm{LE}$ pathway for the positive relevance, and reveals that the essential reasons for determining the decay rate are different. For ${ }^{1} \mathrm{CT} \rightarrow \mathrm{GS}$ pathway, the modulated energy level is the decisive factor. For ${ }^{3} \mathrm{CT} \rightarrow{ }^{3} \mathrm{LE}$ pathway, electronic coupling, which is difficult to modulate, is the dominant factor that determines the decay rate of this process. And it is hard to adjust the loss of the two pathways together through simple molecular design. More importantly, we dig out that the key point limiting PCE is the trade-off in the NFA system with A-DD'D-A structure, gaining a slight of the bottleneck of PCE.

Regardless of experiment or theory, our common appeal is a clear mechanism to improve PCE. The key point is the molecular design strategy. Based on our research, it is speculated that the effects of A-DD'D-A structure have two-sidedness. On the one hand, the modification on D' unit could obtain high-efficiency NFA. On the other hand, it also caused the loss of ${ }^{3} \mathrm{CT}$ state and further restricted PCE. This defect may be compensated by modifying the alkyl chain on the D unit in the A-DA'D-A structure to reduce the driving force and $\Delta E_{3}$, which is currently under study. Another effective method is suppressing the ${ }^{3} \mathrm{CT}$-state loss by promoting charge separation rate is higher than intersystem crossing rate $\left(k_{\mathrm{CS}}>k_{\mathrm{ISC}}\right)$. Although the phenomenon of positive relevance is not promoted, efforts of improving photovoltaic parameters are currently and always underway.

\section{Acknowledgements}

We gratefully acknowledge the financial support from the National Natural Science Foundation of China (Project No. 22163010).

\section{Author Contributions}


Yue Ren: idea, data curation, investigation, manuscripts. Ming-Yang Li: formal analysis, put forward some constructive suggestions for revising the paper. Yun Geng: put forward some constructive suggestions for revising the paper. Yi-Zhou $\mathrm{Xu}$ : data correction and language polishing in major revise. Guang-Yan Sun: funding acquisition, Writing-review \& editing. Ming-Yue Sui: put forward some constructive suggestions for revising the paper. Zhong-Min Su: review and approve manuscripts.

\section{Competing interests}

The authors declare no competing interests.

\section{Additional information}

Supplementary statement of the contents of the models, methods and results, including the detailed excited-state property and each step of the parameters in the equation supplied as Supplementary Information. 


\section{References}

1 Liu, Q. S. et al. 18\% Efficiency Organic Solar Cells. Sci. Bull. 65, 272-275 (2020).

2 Li, C. et al. Non-Fullerene Acceptors with Branched Side Chains and Improved Molecular Packing to Exceed 18\% Efficiency in Organic Solar Cells. Nat. Energy 6, 605-613 (2021).

3 Chen, S. H. et al. High-Performance Polymer Solar Cells with Efficiency over 18\% Enabled by Asymmetric Side Chain Engineering of Non-fullerene Acceptors. Sci. China Chem. 64, 1192-1199 (2021).

4 Yan, C. et al. Non-Fullerene Acceptors for Organic Solar Cells. Nat. Rev. Mater. 3, 18003 (2018).

5 Sun, C. K. et al. Achieving Fast Charge Separation and Low Nonradiative Recombination Loss by Rational Fluorination for High-Efficiency Polymer Solar Cells. Adv. Mater. 31, 1905480 (2019).

6 Li, P. D. et al. Synergistic Effect of Dielectric Property and Energy Transfer on Charge Separation in Non-Fullerene-Based Solar Cells. Angew. Chem. Int. Ed. 60, 15054-15062 (2021).

7 Liu, J. et al. Fast Charge Separation in a Non-fullerene Organic Solar Cell with a Small Driving Force. Nat. Energy 1, 16089 (2016).

8 Zhang, Z. H. et al. Modification on the Indacenodithieno[3,2-b]thiophene Core to Achieve Higher Current and Reduced Energy Loss for Nonfullerene Solar Cells. Chem. Mater. 32, 1297-1307 (2020). 
9 Cui, Y. et al. Over 16\% Efficiency Organic Photovoltaic Cells Enabled by a Chlorinated Acceptor with Increased Open-circuit Voltages. Nat. Commun. 10, 2515 (2019).

10 Qian, D. P. et al. Design Rules for Minimizing Voltage Losses in High-Efficiency Organic Solar Cells. Nat. Mater. 17, 703-709 (2018).

$11 \mathrm{Xu}, \mathrm{Y}$. et al. Tuning the Hybridization of Local Exciton and Charge-Transfer States in Highly Efficient Organic Photovoltaic Cells. Angew. Chem. Int. Ed. 59, 9004-9010 (2020).

12 Eisner, F. D. et al. Hybridization of Local Exciton and Charge-Transfer States Reduces Nonradiative Voltage Losses in Organic Solar Cells. J. Am. Chem. Soc. 141, 6362-6374 (2019).

13 Karki, A., Gillett, A. J., Friend, R. H. \& Nguyen, T.-Q. The Path to 20\% Power Conversion Efficiencies in Nonfullerene Acceptor Organic Solar Cells. $A d v$. Funct. Mater. 11, 2003441 (2021).

14 Chen, X.-K. et al. A unified description of non-radiative voltage losses in organic solar cells. Nat. Energy 6, 799-806 (2021).

15 Rao, A. et al. Exciton Fission and Charge Generation via Triplet Excitons in Pentacene/C60 Bilayers. J. Am. Chem. Soc. 132, 12698-12703 (2010).

16 Wang, R. et al. Nonradiative Triplet Loss Suppressed in Organic Photovoltaic Blends with Fluoridated Nonfullerene Acceptors. J. Am. Chem. Soc. 143, 4359-4366 (2021).

17 Zhang, M. J., Guo, X., Ma, W., Ade, H. \& Hou, J. H. A Large-Bandgap 
Conjugated Polymer for Versatile Photovoltaic Applications with High Performance. Adv. Mater. 27, 4655-4660 (2015).

18 Hess, B., Kutzner, C., van der Spoel, D. \& Lindahl, E. GROMACS 4: Algorithms for Highly Efficient, Load-Balanced, and Scalable Molecular Simulation. J. Chem. Theory Comput. 4, 435-447 (2008).

19 Wang, J. M., Wolf, R. M., Caldwell, J. W., Kollman, P. A. \& Case, D. A. Development and Testing of a General Amber Force Field. J. Comput. Chem. 25, 1157-1174 (2004).

20 Bayly, C. I., Cieplak, P., Cornell, W. \& Kollman, P. A. A Well-Behaved Electrostatic Potential Based Method Using Charge Restraints for Deriving Atomic Charges: the RESP Model. J. Phys. Chem. 97, 10269-10280 (1993).

21 Liu, J. C., Zheng, W. Y., Han, G. C. \& Yi, Y. P. Molecular Origin of CarbonOxygen-Bridge Isomerization Induced Reverse Aggregation Ability in Acceptor-Donor-Acceptor Electron Acceptors for Organic Solar Cells. Sol. RRL 5, 2000780 (2021).

22 Parrinello, M. \& Rahman, A. Polymorphic Transitions in Single Crystals: A New Molecular Dynamics Method. J. Appl. Phys. 52, 7182-7190 (1981).

23 Bussi, G., Donadio, D. \& Parrinello, M. Canonical Sampling Through Velocity Rescaling. J. Chem. Phys. 126, 014101 (2007).

24 Hilborn, R. C. Erratum: "Einstein Coefficients, Cross Sections, f Values, Dipole Moments, and All that" [Am. J. Phys. 50, 982 (1982)]. Am. J. Phys. 51, 471-471 (1983). 
25 Marcus, R. A. On the Theory of Oxidation-eduction Reactions Involving Electron Transfer. I. J. Chem. Phys. 24, 966-978 (1956).

26 Zheng, Z. L., Tummala, N. R., Wang, T. H., Coropceanu, V. \& Brédas, J.-L. Charge-Transfer States at Organic-Organic Interfaces: Impact of Static and Dynamic Disorders. Adv. Funct. Mater. 9, 1803926 (2019).

27 Unger, T. et al. The Impact of Driving Force and Temperature on the Electron Transfer in Donor-Acceptor Blend Systems. J. Phys. Chem. C 121, 22739-22752 (2017).

28 Han, G. C., Guo, Y., Ma, X. Y. \& Yi, Y. P. Atomistic Insight Into Donor/Acceptor Interfaces in High-Efficiency Nonfullerene Organic Solar Cells. Sol. RRL 2, 1800190 (2018).

29 Ernzerhof, M. \& Perdew, J. P. Generalized Gradient Approximation to the Angle- and System-averaged Exchange Hole. J. Chem. Phys. 109, 3313-3320 (1998).

30 Duan, Y.-A. et al. Theoretical Studies on the Hole Transport Property of Tetrathienoarene Derivatives: The Influence of the Position of Sulfur Atom, Substituent and $\pi$-Conjugated Core. Org. Electron. 15, 602-613 (2014).

31 Yanai, T., Tew, D. P. \& Handy, N. C. A New Hybrid Exchange-Correlation Functional using the Coulomb-Attenuating Method (CAM-B3LYP). Chem. Phys. Lett. 393, 51-57 (2004).

32 Lu, T. \& Chen, F. W. Multiwfn: A Multifunctional Wavefunction Analyzer. $J$. Comput. Chem. 33, 580-592 (2012). 
33 Frisch, M. J. et al. Gaussian 09 Rev. D.01, Gaussian, Inc., Wallingford, CT (2009).

34 Vandewal, K., Tvingstedt, K., Gadisa, A., Inganäs, O. \& Manca, J. V. Relating the Open-Circuit Voltage to Interface Molecular Properties of Donor:Acceptor Bulk Heterojunction Solar Cells. Phys. Rev. B 81, 125204 (2010).

35 Chen, X. -K., Wang, T. -H. \& Brédas, J.-L. Suppressing Energy Loss due to Triplet Exciton Formation in Organic Solar Cells: The Role of Chemical Structures and Molecular Packing. Adv. Funct. Mater. 7, 1602713 (2017).

36 Chen, Z. et al. Triplet Exciton Formation for Non-radiative Voltage loss in High-efficiency Nonfullerene Organic Solar Cell. Joule 5, 1832-1844 (2021). 


\section{TOC}

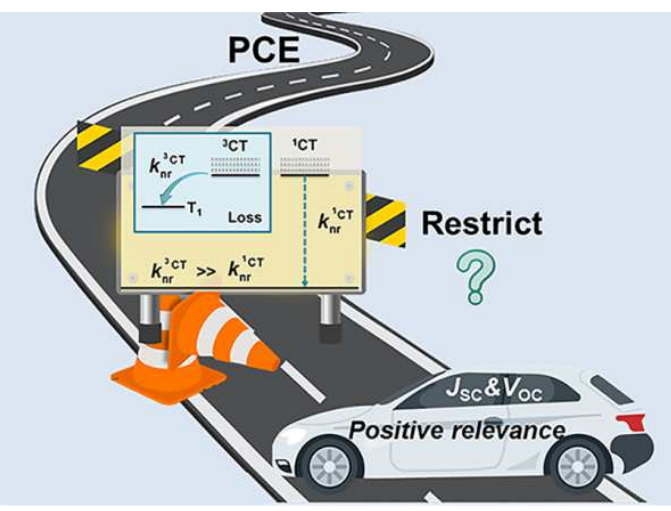


Figures

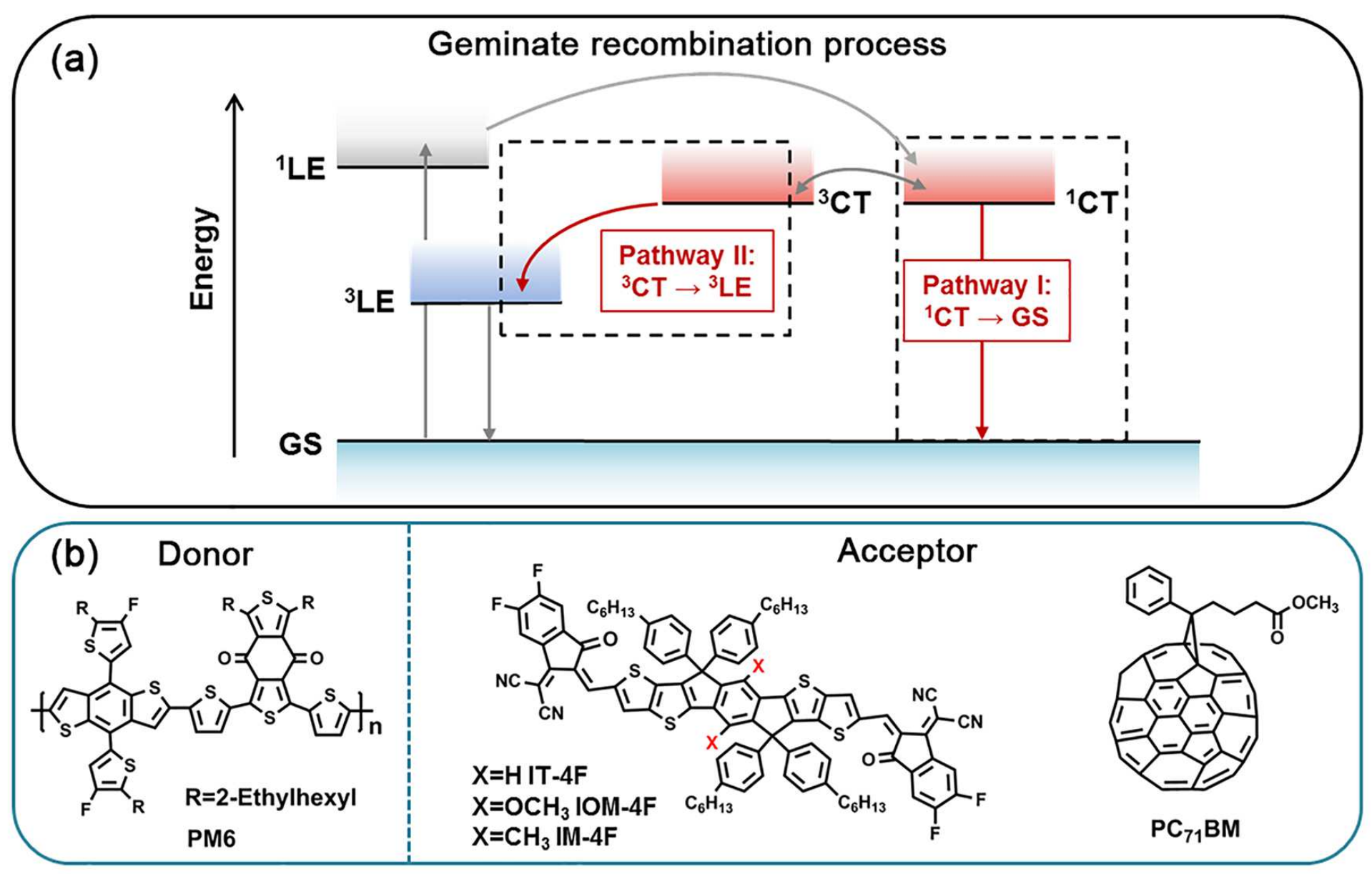

Figure 1

(a) Schematic diagram of geminate recombination process including singlet charge transfer (1CT) state decay to ground state (GS) (1CT囚GS) and triplet charge transfer (3CT) state convert to triplet local exciton (3LE) state (3CT $\varangle \mathrm{LEE}$ ), where $3 \mathrm{CT}$ state is formed by $1 \mathrm{CT}$ state via intersystem crossing. (b) Chemical structures of non-fullerene IT-4F, IOM-4F, IM-4F, fullerene PC71BM as acceptors and PM6 as donors. 


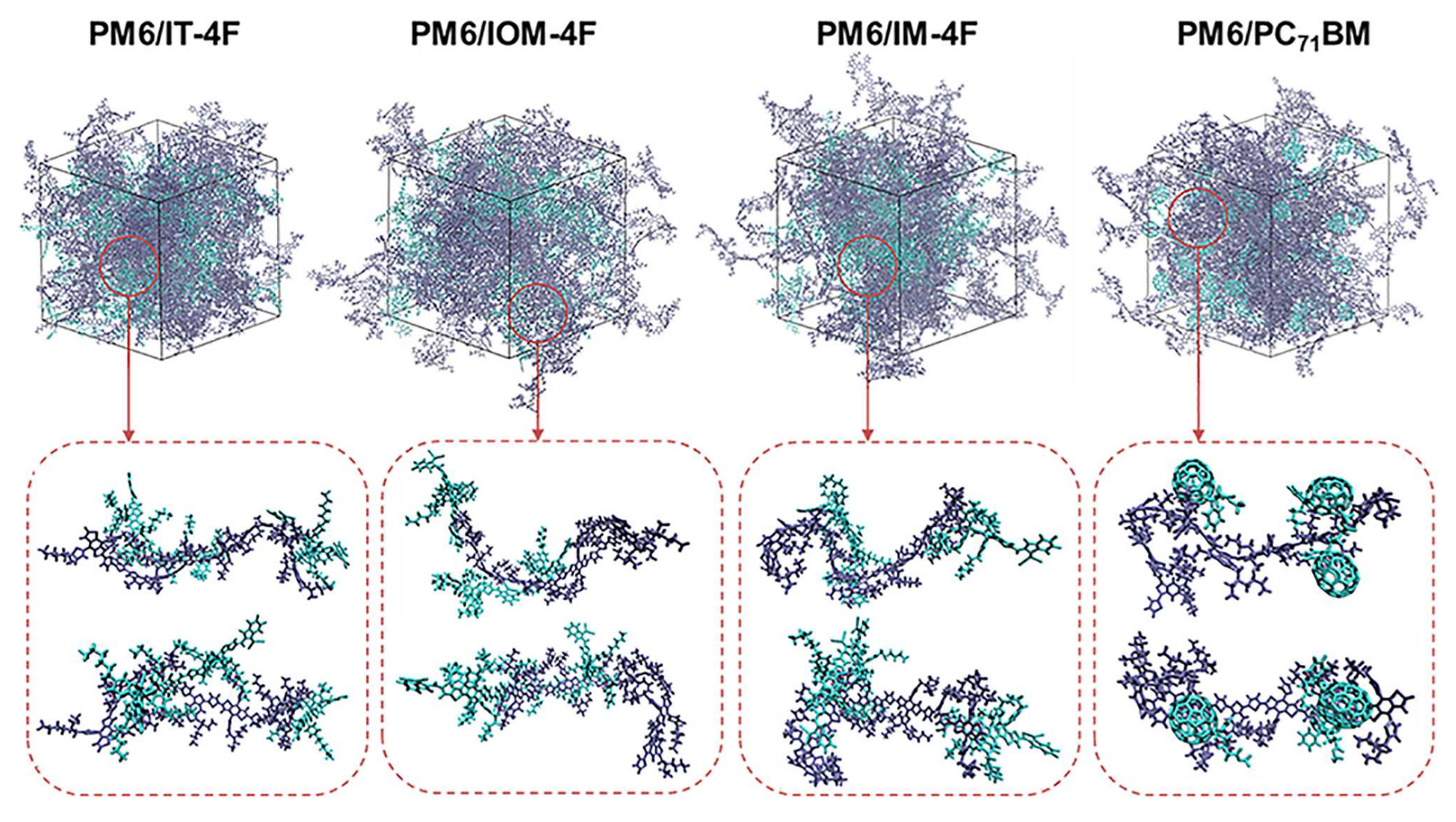

Figure 2

Molecular clusters of PM6/acceptor blends in molecular dynamics (MD) simulations. 


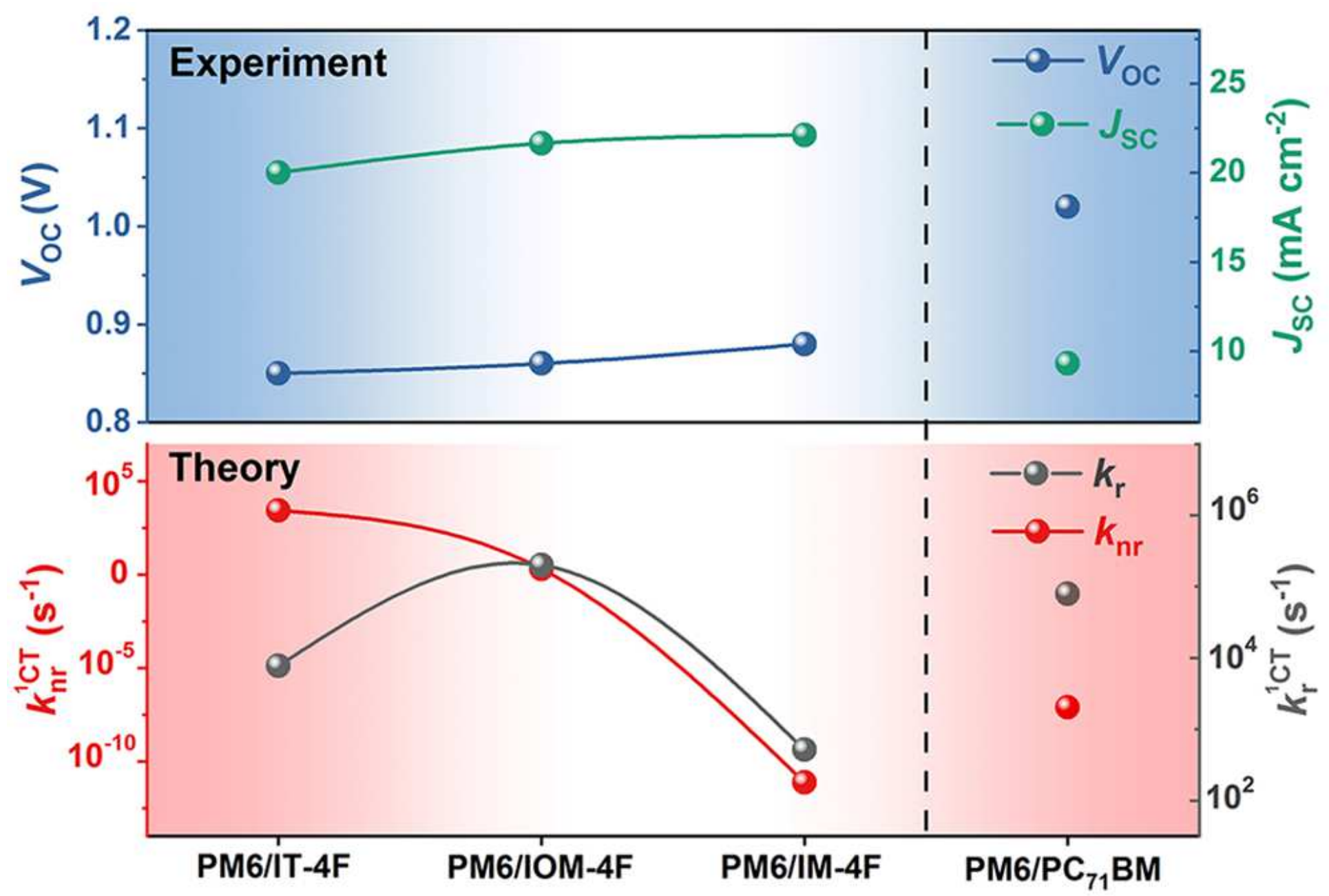

Figure 3

Experimental JSC and VOC and theoretical radiative and non-radiative recombination rates "k" _"r" $\wedge$ "1CT" and "k" _"nr" ^"1CT" of 1CT囚GS pathway (from Ref. 8).

(a)

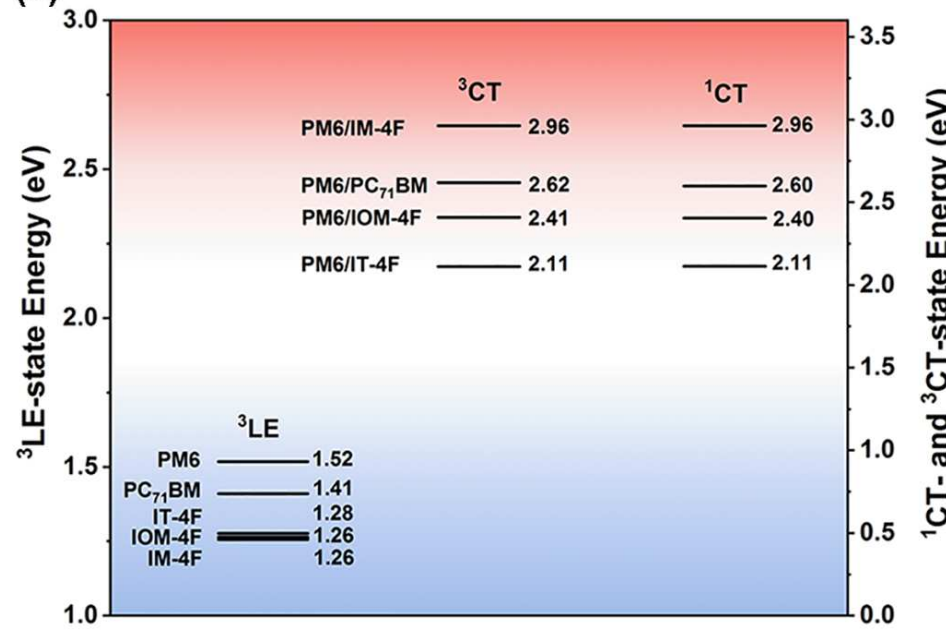

(b)

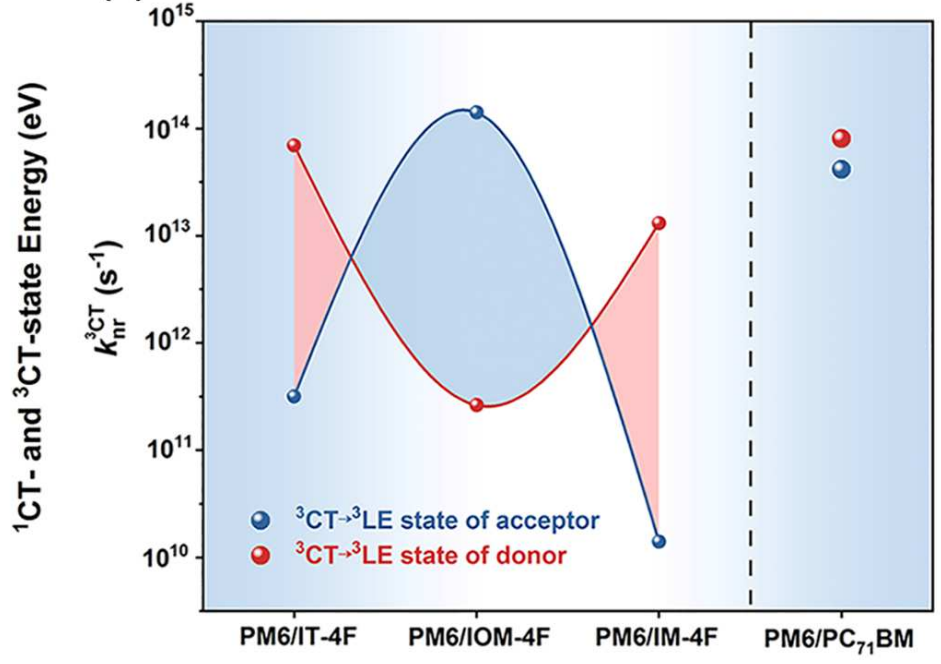

Figure 4 
(a) Schematic diagram of 3LE-states, interfacial 1CT- and 3CT-states energies (eV) for PM6/acceptors blends. (b) The change curve of "k" _"nr" ^"3CT" (s-1), corresponding to decay from 3CT states to 3LE state of donor and acceptors.
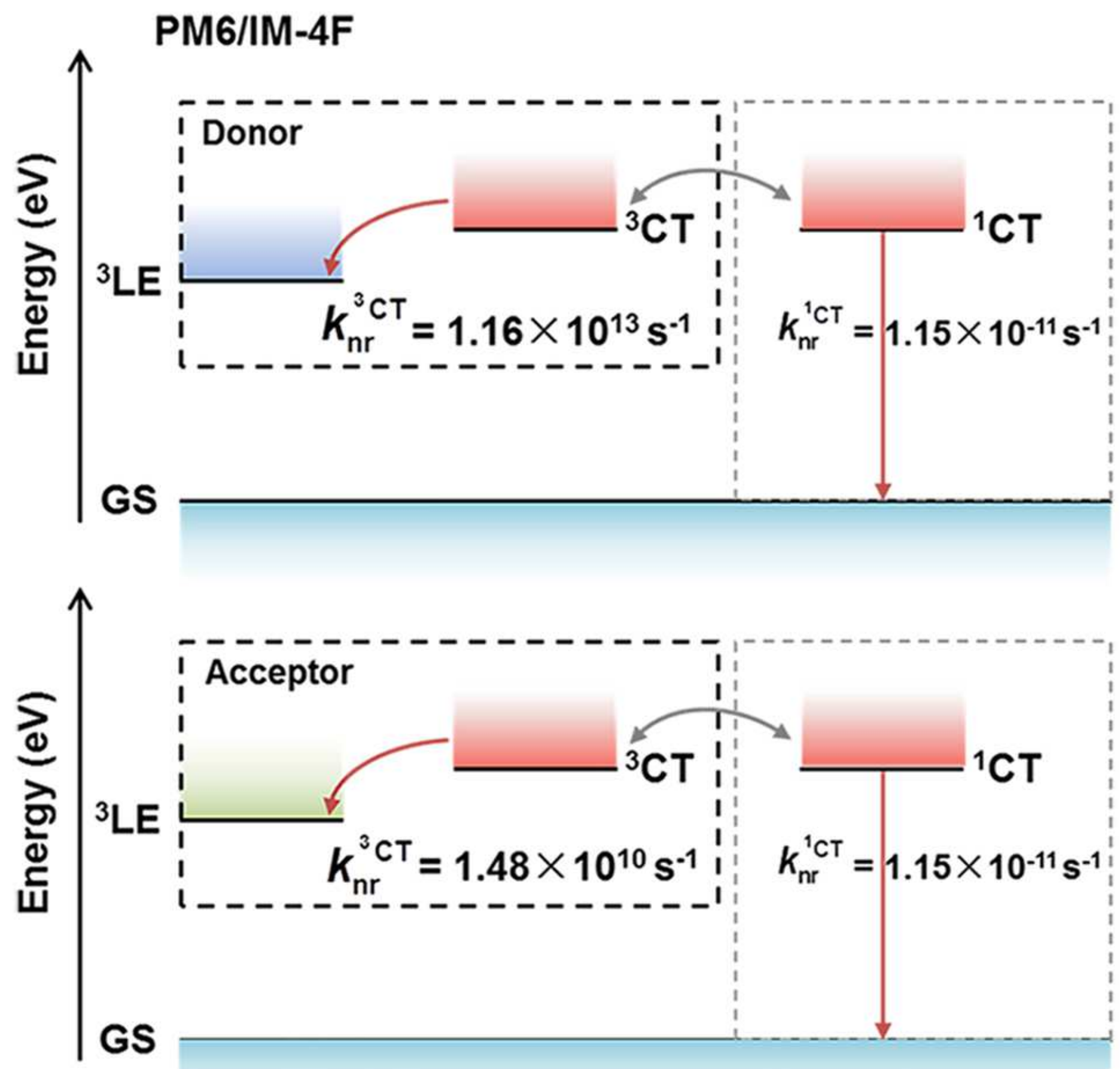

Figure 5

Schematic diagram of non-radiative recombination rates of $1 \mathrm{CT} \otimes \mathrm{GS}$ and $3 \mathrm{CT} \otimes 3 \mathrm{LE}$ pathway in PM6/IM4F blend. The top is the 3CT state decay to 3LE state of donor, and the bottom is decay to 3LE state of acceptor. 
(a)
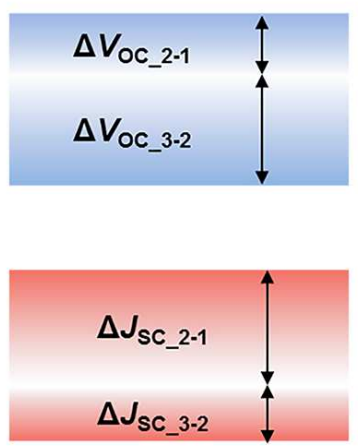

(b) 0

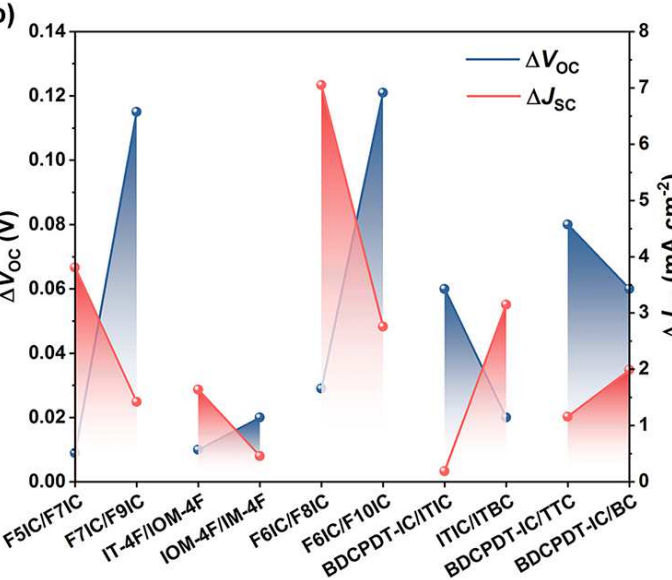

(c)

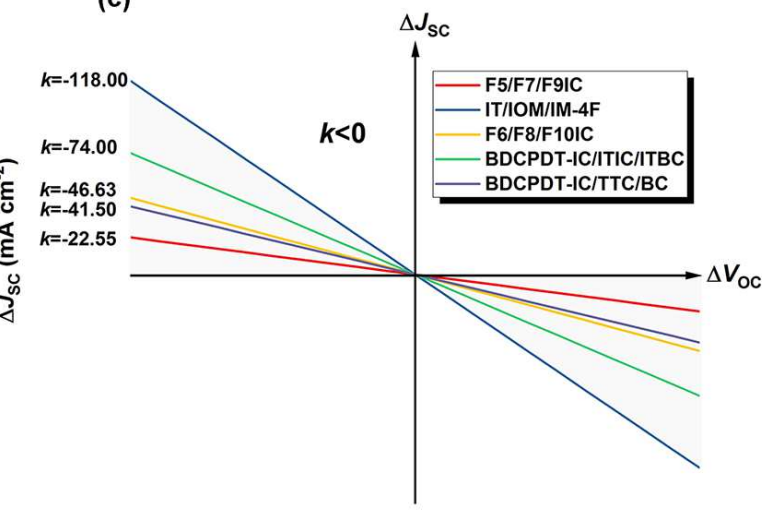

Figure 6

(a) Schematic diagram of increment $\triangle \mathrm{VOC}$ and $\triangle \mathrm{JSC}$ of VOC and JSC measured experimentally, the numeric marker represents the position of the comparison object. (b) Calculated $\triangle \mathrm{VOC}$ and $\triangle \mathrm{JSC}$ for five groups of NFAs, details shown in Supplementary Table 6. (c) Calculated $\triangle \mathrm{JSC}$ as a function of $\triangle \mathrm{VOC}, \mathrm{k}$ is the slope.

\section{Supplementary Files}

This is a list of supplementary files associated with this preprint. Click to download.

- Supplementaryinformation.docx

- TOC.tif 